Article

\title{
A solution of the cosmological constant and DE using breakup of primordial black holes, via a criteria brought up by Dr. Freeze of Austin, Texas, which initiates DE as linked to inflation
}

Andrew Beckwith 1

1 Physics Department, Chongqing University, College of Physics, Chongqing University Huxi Campus, No. 44 Daxuechen Nanlu, Shapinba District, Chongqing 401331, People’s Republic of China, r rwill9955b@gmai.com

Abstract:

We reduplicate the Book "Dark Energy" by M. Li, X-D. Li, and Y. Wang, given zero-point energy calculation with an unexpected "length' added to the 'width' of a graviton wave just prior to specifying the creation of 'gravitons', while using Karen Freeze's criteria as to the breakup of primordial black holes to give radiation era contributions to GW generation. The GW generation will be when there is sufficient early universe density so as to break apart Relic Black holes of the order of Planck mass ( $10^{\wedge} 15$ grams) which is about when the mass of relic black holes is created, $10^{\wedge}-27$ or so seconds after expansion starts. Needles to state a key result will be in the initial potential V calculated, in terms of other input variables

Keywords: Minimum scale factor, cosmological constant, DE, Arrow of time

\section{Introduction}

What we are doing is to try to confirm if we can apply the techniques of the following reference to the problem of DE and the arrow of time, and heavy gravity. After work I did in [1] was allegedly not credible, due to people having doubts as to the existence of a multiverse and equating two first integrals as I did, via early pre Planckian space-time, the following reference was accessed [2]. And then applied to [3] and the work on heavy gravity in [4]. In doing so we will keep in mind the $\mathrm{t}^{\prime}$ Hooft memorandum as to the arrow of time, which is in [5] as a basic organizational principle for our discussion, i.e. formation of our program is assuming initial conditions for using [4] in the expansion of the universe say after $10^{\wedge}-42$ seconds

$$
m_{g}=\frac{\hbar \sqrt{\Lambda}}{c}
$$

This release of conditions for massive gravity should be in line with what is in Freeze

et.al.

$$
\rho_{B H}=\frac{m_{p}^{4}}{32 \pi} \cdot\left(\frac{m_{P}}{m}\right)^{4} \cdot \frac{1}{\left|1+3 w_{Q}\right|}
$$

If the conditions of an early universe, are greater than this value, for Eq.(2)[6] then according to Freeze, et.al. primordial black holes would break apart. We state that this break up of primordial black holes would be enough to create an initial "sea" of gravitons, due to Eq. (1) which would then add up to be in effect a value for a sufficient number of early universe gravitons, which would be added up per unit volume, to in fact sum up to an energy density equivalent to Eq.(1) so we have massive gravitons and DE. Hence we will be adding up the number of gravitons which may be released due to Eq. (2) and [7] which states the number of gravitons which may be emitted due to a black hole as given in it's page 47 is 
.1 percent of emitted energy from a nonrotating black hole. Keep in mind that this is for black holes, as given in [7] with mass

$$
M_{\text {black-hole-primordial }} \sim 10^{15} \times\left(\frac{t}{10^{-23} \mathrm{~s}}\right) \cdot g(\text { grams })
$$

For a $10^{\wedge}-5$ gram black hole, $\mathrm{t}$ would have to be about $10^{\wedge}-33$ seconds, and according to inflation expands space by a factor of $10^{\wedge} 26$ over a time of the order of $10^{\wedge}-33$ to $10^{\wedge}-32$ seconds. Meaning we had $10^{\wedge}-5$ gram black holes at the end of inflation, and at the time the density of space would be greater than Eq. (2) we would have a breakup of black holes if we had space-time density greater than or equal to about Eq. (2) then we have $.1 \%$ of the mass of the broken $\mathrm{BH}$ contributing to gravitons, which after we review it may be relevant to Eq. (1) above. One Planck mass is about $10^{\wedge}-5$ grams. And it is worth noting in our development when we go past inflation, that we have Black holes growing to the value of about $10^{\wedge} 11$ grams, after $10^{\wedge}-27$ seconds which is for a radii of approximately $10^{\wedge} 6$ kilometers, whereas we can and will define Black holes of $10^{\wedge}-5$ grams which would be for less than a centimeter radii just after the end of inflation.

The discussion below will reflect these values and put them in the context of answering how the breakup of Blackholes, due to the phenomenon brought up in [7] allows for the production of gravitons which we conflate with DE. The radius of the inflationary universe at $10^{\wedge}-33$ seconds would be about $10^{\wedge}-3$ centimeters to at most $10^{\wedge}-1$ centimeters. If it was the latter, we have

$$
\begin{gathered}
a(t)=a_{\text {initial }} t^{\gamma} \\
\rho \approx \frac{\dot{\phi}^{2}}{2}+V(\phi) \equiv \frac{\gamma}{8 \pi G} \cdot t^{2}+V_{0} \cdot\left\{\sqrt{\frac{8 \pi G V_{0}}{\gamma(3 \gamma-1)}} \cdot t\right\} \sqrt{\frac{\gamma}{4 \pi G}}-\sqrt{\frac{8 \pi G}{\gamma}} \\
P_{G}=\frac{\gamma \cdot t^{2}}{8 \pi G}-V_{0} \cdot\left\{\frac{\sqrt{8 \pi G V_{0}}}{\gamma \cdot(3 \gamma-1)} \cdot t\right\}^{\sqrt{\frac{\gamma}{4 \pi G}}-\sqrt{\frac{8 \pi G}{\gamma}}} \\
\rho_{B H}=\frac{m_{p}^{4}}{32 \pi} \cdot\left(\frac{m_{P}}{m}\right)^{4} \cdot \frac{1}{\left|1+3 \frac{P_{G}}{\rho_{G}}\right|}
\end{gathered}
$$

What we will be looking at would be having a glimpse of 


$$
\frac{P_{G}}{\rho_{G}} \approx \frac{\frac{\gamma \cdot t^{2}}{8 \pi G}-V_{0} \cdot\left\{\frac{\sqrt{8 \pi G V_{0}}}{\gamma \cdot(3 \gamma-1)} \cdot t\right\}^{\sqrt{\frac{\gamma}{4 \pi G}}-\sqrt{\frac{8 \pi G}{\gamma}}}}{\frac{\gamma \cdot t^{2}}{8 \pi G}+V_{0} \cdot\left\{\frac{\sqrt{8 \pi G V_{0}}}{\gamma \cdot(3 \gamma-1)} \cdot t\right\}^{\sqrt{\frac{\gamma}{4 \pi G}}-\sqrt{\frac{8 \pi G}{\gamma}}}}
$$

Doing this would be at a minimum of having, a distance greater than or equal to

$$
\lambda_{D E} \approx 10^{30} \ell_{\text {Planck }}
$$

Assume then we have, for the sake of argument

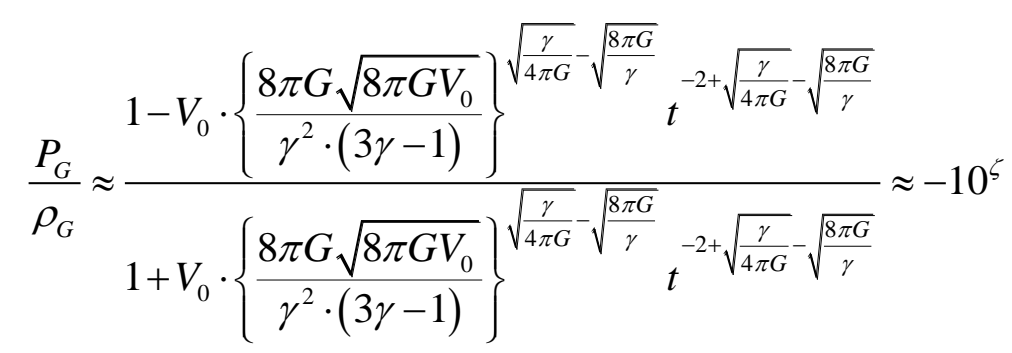

The particulars of the coefficient of the right hand side of Eq.(10), if $\hbar=G=\ell_{P}=t_{P}=1$, then if we set

$$
\frac{\gamma}{4 \pi}-2 \sqrt{\frac{\gamma}{4 \pi}}-1=0
$$

So then we have if we wish to neutralize senstivity to time itself at first approximation

$$
\gamma=4 \pi \cdot(1 \pm \sqrt{2})^{2}
$$

We can then look at the following

$$
\frac{1-V_{0} \cdot\left\{\frac{8 \pi \sqrt{8 \pi V_{0}}}{16 \pi^{2} \cdot(1 \pm \sqrt{2})^{4} \cdot\left(12 \pi \cdot(1 \pm \sqrt{2})^{2}-1\right)}\right\}^{(1 \pm \sqrt{2})-\frac{\sqrt{2}}{(1 \pm \sqrt{2})}}}{1+V_{0} \cdot\left\{\frac{8 \pi \sqrt{8 \pi V_{0}}}{16 \pi^{2} \cdot(1 \pm \sqrt{2})^{4} \cdot\left(12 \pi \cdot(1 \pm \sqrt{2})^{2}-1\right)}\right\}^{(1 \pm \sqrt{2})-\frac{\sqrt{2}}{(1 \pm \sqrt{2})}} \approx-10^{\zeta}}
$$

The above equation can be used, to locate appropriate values for $V_{0}$ in units where $h=G=\ell_{P}=t_{P}=m_{P}=c=1$

Given an approximate value for $V_{0}$ we will then proceed to come up with examining 


$$
\begin{gathered}
\frac{P_{G}}{\rho_{G}} \approx \frac{1-V_{0} \cdot\left\{\frac{8 \pi G \sqrt{8 \pi G V_{0}}}{\gamma^{2} \cdot(3 \gamma-1)}\right\}^{\sqrt{\frac{\gamma}{4 \pi G}}-\sqrt{\frac{8 \pi G}{\gamma}}} t^{-2+\sqrt{\frac{\gamma}{4 \pi G}}-\sqrt{\frac{8 \pi G}{\gamma}}}}{1+V_{0} \cdot\left\{\frac{8 \pi G \sqrt{8 \pi G V_{0}}}{\gamma^{2} \cdot(3 \gamma-1)}\right\}^{\sqrt{\frac{\gamma}{4 \pi G}}-\sqrt{\frac{8 \pi G}{\gamma}}} t^{-2+\sqrt{\frac{\gamma}{4 \pi G}}-\sqrt{\frac{8 \pi G}{\gamma}}}} \\
\rho_{B H}=\frac{m_{p}^{4}}{32 \pi} \cdot\left(\frac{m_{P}}{m}\right)^{4} \cdot \frac{1}{\left|1-3 \cdot 10^{-\zeta}\right|} \\
M_{\text {black-hole-primordial }} \sim 10^{15} \times\left(\frac{t}{10^{-23} s}\right) \cdot g(\text { grams })
\end{gathered}
$$

Whereas we ask for initial conditions for the arrow of time, and $\Lambda$ and DE formation once we understand what happens if Eq. (14) is exceeded by the initial density of the early universe.

\section{Reviewing conditions for the early universe, assuming a volume of inflation at its end and the beginning of radiation domination to employ Eq. (15) depending upon what is put into $m$ at $t \approx 10^{-33} \mathrm{~s}$}

The easiest solution to Eq. (14) is to look at when $\zeta=0$ when we have Dark Energy, and then Look at when an object of mass $\mathrm{m}$, and radius $\mathrm{R}$ is pulled apart. From [7], page 154

$$
m \approx \frac{8 \pi R^{3}(\text { radius }) \cdot \rho}{3}
$$

With this generalized to being for black holes being pulled apart when

$$
\begin{aligned}
& m \approx-\left(\frac{4 \pi \rho}{3}\right) \cdot\left(1+3 \cdot \frac{P_{G}}{\rho_{G}}\right) \cdot \frac{8 m^{3}}{m_{P}^{6}} \\
& \Rightarrow \text { if } P_{G} \approx-\rho_{G} \\
& m(\text { black}- \text { holes }) \geq 10^{5} m_{P} \quad \text { break-apart } \\
& \text { after } t \approx 10^{-27} \mathrm{sec}
\end{aligned}
$$

We then have, say that there are a large number of black holes of about $10^{\wedge} 5$ Planck Mass at a time just after the end of the inflationary era, which are pulled apart, and if we then look at the formula of $1 / 1000$ of the mass of a black hole we are talking about a contribution of about $10^{2} m_{P}$ in black hole generation of a flux of gravitons after $10^{\wedge}-27$ seconds, i.e. for the regime from $10^{\wedge}-33$ seconds to $10^{\wedge}-27$ seconds, we get

$$
10^{2} m_{P}(\text { gravitons }) \Rightarrow 10^{67} \text { gravitons per } 10^{5} m_{P}(\text { black-hole })
$$

3. Making DE equivalent to a sea of initial gravitons, in regime $10^{\wedge}-33$ to $10^{\wedge}-27$ seconds Roughly put, one hydrogen atom is about 1.66 times $10^{\wedge}-24$ grams. The weight of a 
Massive graviton is about $10^{\wedge}-65$ grams [8][9], hence we are talking about $10^{\wedge}-22$ grams, or about $10^{\wedge} 44$ gravitons, with each graviton about $6 \times 10^{-32} \mathrm{eV} / \mathrm{c}^{2}$ After $10^{\wedge}-27$ seconds, the following in the set of equations given below are Equivalent, and that these together will lead to a cosmological Constant, $\Lambda$ of the sort which we will be able to refer to later

$$
1 \text { graviton } \approx 10^{-65} \mathrm{~g}
$$

$$
M_{P B H} \sim 10^{15} \times\left(\frac{t=10^{-27} s}{10^{-23} s}\right) \cdot g(\text { grams }) \approx 10^{11} g \approx 10^{16} m_{P}
$$

Assuming that gravitons contribute to the Dark Energy value will lead to us using the Karen Freeze model, with gravitons being released in the early universe by the breakup of early universe black holes which have a maximum value of about $10^{\wedge} 11 \mathrm{~g}$, as opposed to the value of the Sun which has about $10^{\wedge} 33$ grams, i.e. by making use of the following

$D E($ from-broken black hole $) \sim 7 \times 10^{-30} \mathrm{~g} / \mathrm{cm}^{3} \approx 7 \times 10^{12} \mathrm{~g} /\left(10^{9} \mathrm{~km}\right)^{3}$

Universe $($ black hole $) \sim 7 \times 10^{15} \mathrm{~g} /\left(10^{9} \mathrm{~km}\right)^{3} \approx 7 \times 10^{4} M_{P B H} /\left(10^{9} \mathrm{~km}\right)^{3}$

We claim that the above Eq.(17c), will be able to yield a DE value $\Leftrightarrow 10^{80}$ gravitons in a region of space for which we have a "sphere" of radius $10^{\wedge} 9 \mathrm{~km}$ at $10^{\wedge}-27$ seconds as a way to support the existgence of the mass of DE. i.e. about $10^{\wedge} 4$ number of black holes given in Eq.(17d) initially. And now we can look at the to the calculation given by the following as by [3]

$$
\begin{aligned}
& \frac{1}{2} \cdot \sum_{i} \omega_{i} \equiv V(\text { volume }) \cdot \int_{0}^{\hat{\lambda}} \sqrt{k^{2}+m^{2}} \frac{k^{2} d k}{4 \pi^{2}} \approx \frac{\hat{\lambda}^{4}}{16 \pi^{2}} \\
& \underset{\hat{\lambda}=M_{\text {Planck }}}{\longrightarrow} \rho_{\text {boson }} \approx 2 \times 10^{71} G e V^{4} \approx 10^{119} \cdot\left(\rho_{D E}=\frac{\Lambda}{8 \pi G}\right)
\end{aligned}
$$

In stating this we have to consider that

$$
\rho_{D E}=\frac{\Lambda}{8 \pi G} \approx \hbar \cdot \frac{(2 \pi)^{4}}{\lambda_{D E}^{4}}
$$

so then that we can consider we have after $10^{\wedge}-27$ seconds

$$
\lambda_{D E} \approx 10^{30} \ell_{\text {Planck }}
$$

We then have to consider how to reach the experimental conditions for when 


$$
\rho_{D E}=\frac{\Lambda}{8 \pi G} \approx \hbar \cdot \frac{(2 \pi)^{4}}{\lambda_{D E}^{4}}
$$

After we fill in more details of this procedure, we will be considering the implications of the paper by Alves [11] which conflates DE with gravitons which is the approach we are using and the idea is that one uses gravitons in say a sphere of about 1 million kilometers to give the same energy "density" as gravitons in a way to recover the DE density. From here, we should realize that Eq. (19) is commensurate with a "length" within a few orders of magnitude similar to a centimeter, making the above "analysis" similar to DE being formed at the start near the end of inflation.

\section{What about representative wavefunctions within the radii of $10^{\wedge}--3$ centimeters to $10^{\wedge} 6$ kilometers?}

The cleanest representation the author found is given in [12] where on page 47 the Hartle -Hawking Wavefunction for minisuperspace cosmology, with a no boundary condition would lead to, if $a^{2}(t) \cdot V(\phi(t))<1$, a value of, if $V(\phi(t))$ is defined by

$$
V(\phi(t))=V_{0} \cdot\left(\sqrt{\frac{8 \pi G V_{0}}{v \cdot(3 v-1)}} \cdot t\right)^{\frac{1}{2} \cdot \sqrt{\frac{v}{\pi G}}-4 \sqrt{\frac{\pi G}{v}}}
$$

Then, use from [12] a saddle point solution to the Wheeler De Witt equation of the form

$$
\Psi_{H H}(a, \phi(t)) \approx \exp \left[\frac{1}{3 V(\phi(t))}\right] \times \exp \left[\frac{-\left(1-a^{2}(t) \cdot V(\phi(t))\right)}{3 V(\phi(t))}\right]
$$

At inflation, $\mathrm{z}$ (red shift) $10^{\wedge}-25$, implying a tiny scale factor so Eq.(22) would work.

Having said that, the term $V_{0}$ cold be set by Eq. (13) especially, and the main work for future work would be comparing Eq. (22) with the so called wavefunction of a black hole as given by [13], by

$$
\begin{aligned}
& \Psi_{B H} \approx P \cdot \exp (-4 \pi M) \exp \left(-I_{2}\right) \\
& M \approx \text { mass }- \text { of }- \text { black }- \text { hole }- \text { plus - background } \\
& I_{2}=\text { dynamics }- \text { of }- \text { local }- \text { deg-of }- \text { freedom } \\
& \approx \frac{1}{2} \cdot \sum_{\chi} \omega_{\chi} \cdot\left\{\tanh \left(\beta \omega_{\chi} / 4\right) f_{\chi,+}^{2}+\frac{f_{\chi,-}^{2}}{\tanh \left(\beta \omega_{\chi} / 4\right)}\right\}
\end{aligned}
$$

Here, we have that we can also look at [14] where we can look at

$$
\begin{aligned}
& S_{B H} \approx k_{B} \tilde{A} / 4 \ell_{P}=k_{B} M^{2} G^{2} / c^{4} \\
& R_{\text {Schwartzshild }}=2 M G / c^{2}
\end{aligned}
$$

If $\mathrm{M}$ is $\sim 10^{\wedge}-18$ solar mass, about $10^{\wedge} 15$ grams, it means that the dynamics of the local degrees of freedom of the black hole [15] that the approximation used is that exists 


$$
\begin{aligned}
& \mathrm{A}_{n}=4 \cdot(\ln 3) \cdot n_{\text {quantum }} \\
& \mathbb{N}=(\ln 3) \cdot \mathrm{A}_{n} \\
& M=\mathbb{N} \cdot(\ln 3) \cdot \frac{T}{2} \\
& T=(h / 2 \pi) c^{3} /(8 \pi G M k)
\end{aligned}
$$

Then by Eq,. (24) we can write out the following

$$
\begin{aligned}
& T_{B H}=(h / 2 \pi) c^{3} /(8 \pi G M k) \approx 10^{12} \text { kelvin } \\
& \text { if } M_{B H} \approx 10^{15} \mathrm{~g}, \text { or } 10^{-18} M_{\text {Sun }}
\end{aligned}
$$

IF this is true, then we have the situation, where the Black holes being broken apart by the reasons given in [7] would lead to , in first order an effective black hole wavefunction approximately set and looking like, when $M_{B H} \approx 10^{15} \mathrm{~g}$, or $10^{-18} M_{\text {Sun }}$ that an individual black hole wavefunction is,

$$
\Psi_{B H} \approx P \cdot \exp (-4 \pi M)
$$

With $M_{B H} \approx 10^{15} \mathrm{~g}$, or $10^{-18} M_{\text {Sun }}$ assumed to be equivalent to Eq. (24c), and then $\mathrm{M}$ has data from Degrees of freedom already included.

This value of Eq. (26) needs to be compared to, if we have say $10^{\wedge} 4$ or so black holes being broken up in the first $10^{\wedge}-27$ seconds in order to get DE according to Eq.(20) and Eq. (17c), and Eq.(26) would be for each of the up to $10^{\wedge} 4$ black holes releasing gravitons, witb perhaps a maximum value of up to $10^{\wedge} 80$ gravitons released as to obtain a mass- energy value for $\mathrm{DE}$ which should be equivalent to Eq. (18a) and Eq.(19).

Meanwhile, the up to $10^{\wedge} 4$ black holes represented are such that conceivably a relationship between Eq. (13) and $V_{0}$ exists, so then that one can identify, using Planckian units of the following type $\hbar=G=\ell_{P}=t_{P}=m_{P}=c=1$ a "magnitude" of $V_{0}$ exists, so we can the examine if there a Relationship linkage as follows

A. Specify $V_{0}$ as given above, for a given time $t \sim 10^{\wedge} 27$ seconds.

B. Use that to give a value, then try to satisfy this criteria this inhomogenity requirement of[16]

$$
\frac{H^{2}}{\dot{\phi}} \approx \sqrt{\frac{4 \pi G}{v}} \cdot V_{0} \cdot\left(\sqrt{\frac{8 \pi G V_{0}}{v \cdot(3 v-1)}}\right)^{\frac{1}{2} \cdot \sqrt{\frac{v}{\pi G}}-4 \sqrt{\frac{\pi G}{v}}} t^{\frac{1}{2} \cdot \sqrt{\frac{v}{\pi G}}-4 \sqrt{\frac{\pi G}{v}}+1} \approx 10^{-5}
$$

One can ssoluate out a value of the coefficient of expansion using this, for $\gamma$ as used in Eq.(4). 
C. After we have this value satisfied, set

$$
\begin{aligned}
& 1-V_{0} \cdot\left\{\frac{\left.8 \pi G \sqrt{8 \pi G V_{0}}\right)}{\gamma^{2} \cdot(3 \gamma-1)}\right\} \sqrt{\frac{\gamma}{4 \pi G}}-\sqrt{\frac{8 \pi G}{\gamma}} t^{-2+\sqrt{\frac{\gamma}{4 \pi G}}-\sqrt{\frac{8 \pi G}{\gamma}}} \\
& \approx-10^{\zeta} \cdot\left(1+V_{0} \cdot\left\{\frac{8 \pi G \sqrt{8 \pi G V_{0}}}{\gamma^{2} \cdot(3 \gamma-1)}\right\}^{\sqrt{\frac{\gamma}{4 \pi G}}-\sqrt{\frac{8 \pi G}{\gamma}}} t^{-2+\sqrt{\frac{\gamma}{4 \pi G}}-\sqrt{\frac{8 \pi G}{\gamma}}}\right)
\end{aligned}
$$

D. Use this, if $\zeta$ set small to isolate out values of frequency for which we can have at $t \sim 10^{\wedge}-27 \mathrm{~s}$

$$
\begin{aligned}
& V=V_{0} \cdot\left\{\sqrt{\frac{8 \pi G V_{0}}{\gamma(3 \gamma-1)}} \cdot t\right\}^{\sqrt{\frac{\gamma}{4 \pi G}}-\sqrt{\frac{8 \pi G}{\gamma}}} \\
& \underset{l_{\text {Planck }} \equiv \hbar \equiv G \equiv 1}{\longrightarrow} V_{0} \cdot\left\{\sqrt{\frac{8 \pi V_{0}}{\gamma(3 \gamma-1)}} \cdot t\right\}^{\sqrt{\frac{\gamma}{4 \pi}}-\sqrt{\frac{8 \pi}{\gamma}}} \approx \omega \propto\left(\frac{2}{3 a_{\text {min }}}\right)^{-1 / \gamma}
\end{aligned}
$$

This frequency value, say of radiation will be to look at the frequency range of DE, with Eq. (29) allowing for inputs of $V(\phi(t))$ of Eq.(29) into Eq. (22), with $V(\phi(t)) \approx \omega_{D E}$

E. Afterwards, with this value of frequency, examine

$$
\rho \approx \frac{\dot{\phi}^{2}}{2}+V(\phi) \equiv \frac{\gamma}{8 \pi G} \cdot t^{2}+V_{0} \cdot\left\{\sqrt{\frac{8 \pi G V_{0}}{\gamma(3 \gamma-1)}} \cdot t\right\}^{\sqrt{\frac{\gamma}{4 \pi G}}-\sqrt{\frac{8 \pi G}{\gamma}}}
$$

Whereas the frequency is for, roughly looking at what we will be thinking of if we put in Eq.(12) when $\mathrm{G}=1=\hbar$ is: for a time between $\mathrm{t}$ set as $10^{\wedge}-33$ seconds to $10^{\wedge}-27$ seconds

$$
\begin{aligned}
& \rho \approx \frac{\gamma t^{2}}{8 \pi G}+V_{0} \cdot\left\{\frac{\sqrt{8 \pi G V_{0}}}{\gamma^{2} \cdot(3 \gamma-1)}\right\}^{\sqrt{\frac{\gamma}{4 \pi G}}-\sqrt{\frac{8 \pi G}{\gamma}}} t^{\sqrt{\frac{\gamma}{4 \pi G}}-\sqrt{\frac{8 \pi G}{\gamma}}} \\
& \approx \frac{t^{2}}{\sqrt{2}}+V_{0} \text { if } \quad \gamma=\frac{8 \pi}{\sqrt{2}}
\end{aligned}
$$

At $\quad t \sim 10^{\wedge} 17$ times Planck time, the first term is overshadowed by $V_{0}$ with $V_{0}$ proportional to the frequency $\omega$ where this can be for gravitons emitted from black holes of mass $10^{\wedge} 15 \mathrm{~g}$, or initially having wavelength $\lambda_{D E} \approx 10^{30} \ell_{\text {Planck }}$, which corresponds to frequency about $10^{\wedge} 13 \mathrm{~Hz}$, which if we look at the following relation, namely 
$\left(1+z_{\text {Radii } 1 \text { billion-km }}\right) \omega_{\text {Earth-orbit }} \approx z_{\text {Radii } 1 \text { billion-km }} \omega_{\text {Earth-orbit }} \approx \omega_{\text {Radii } \sim \text { billion-km }} \propto 10^{4} \mathrm{GHz}$

and

$$
z_{\text {Radii } 1 \text { billion-km }} \approx 10^{4} \Rightarrow \omega_{\text {Earth-orbit }} \approx 1 G H z
$$

The figure of red shift about $\mathrm{z} \sim 10^{\wedge} 4$ for a radii of about one billion kilometers at $\mathrm{t} \sim 10^{\wedge}-27 \mathrm{~s}$ is to be seen and compared to $z \sim 1100$ about 100,000 years after the start of the inflationary era. Point in fact being that at the end of inflation, that acceleration ceased, for a time with the zero value of acceleration reached at $10^{\wedge}-32-10^{\wedge} 33$ seconds, so then the one billion $\mathrm{km}$ at $\mathrm{t} \sim 10^{\wedge}-27 \mathrm{~s}$ represents a slowing down of the rate of expasion of the universe, whereas $z^{\sim} 10^{\wedge} 25$ at the end of inflation wheras $\mathrm{z}$ drops 21 orders of magnitude to $\mathrm{z} \sim 10^{\wedge} 4$ at about $\mathrm{t} \sim 10^{\wedge}-27 \mathrm{~s}$ whereas $\mathrm{z}$ futher "shrinks" to about 1100 , or roughly $10^{\wedge} 3$ at about $\mathrm{t} \sim 100,000$ years

\section{What can be inferred as to subsequent growth of entropy from early times to today?}

At about $\mathrm{z} \sim 10^{\wedge} 4$, at about time $\sim 10^{\wedge}-27$ seconds we have about $10^{\wedge} 80$ gravitons, and from here[17]

$$
S(k)=\ln \left(\Gamma / \Gamma_{0}\right)=r_{k}+\ln 2+1 / 2 \ln n(k) \propto 10^{80}
$$

The value of the "final" phase state, $\Gamma$ would be the "final" phase state, about $10^{\wedge}-27 \mathrm{~s}$ after inflation

The value of the "initial" phase state, $\Gamma_{0}$ would be the phase state, about $10^{\wedge}-32$ after inflation

The ratio would be due to $\Gamma=17,621117.2689$ "bigger" than $\Gamma_{0}$, whereas we would scale

$$
\left(\Gamma / \Gamma_{0}\right) \approx 1.762 \times 10^{8}
$$

This is the ratio of expansion of the "graviton" based entropy, forming a value of DE which may be commensurate with the number of gravitons released in a volume of space, from radius of 1 centimeter to a radius of 1 times $10^{\wedge} 9$ kilometers.

As from $\mathrm{z} \sim 0$, at about 13.8 billion years $\sim 43,5485937613.56$ times $10^{\wedge} 16$ seconds to $10^{\wedge}-32$ seconds

The value of $\Gamma_{\text {Today }}$ would be $t$ he Today's phase state at $43,5485937613.56$ times $10^{\wedge} 16$ seconds

The value of the "initial" phase state, $\Gamma_{0}$ would be the phase state, about $10^{\wedge}-32$ after inflation

$$
S_{\text {Today }}(k)=\ln \left(\Gamma_{\text {Today }} / \Gamma_{0}\right) \propto 10^{105}(36)
$$

For what it is worth, the radius of the universe, TODAY is about $8.8 \times 10^{23}$ kilometers

From about $10^{\wedge} 9$ kilometers at $10^{\wedge}-27$ seconds, and about .0001 kilometers at $10^{\wedge}-32$ seconds

i.e. roughly $10^{\wedge} 28$ times the radial distance larger than the value of the radius at end of inflation. And, if have this baseline, we can compare it against purely thermal treatments of cosmology

5. Examining what was done by Rosen, in 1991, which had some fidelity with the 


\section{same issues with the idea of using a breakup of black holes, for DE}

The key point of this mini chapter will be to summarize the derivation of the temperature[2]

$$
T=\left(\rho_{P} / \sigma\right)^{1 / 4} \cdot \frac{\vec{a} r^{7}}{\left(\breve{a}^{4}+r^{4}\right)^{2}}
$$

Whereas $\left(\rho_{P} / \sigma\right)^{1 / 4}=1.574 \times 10^{32} K($ kelvin $)$, and $\breve{a}=10^{-3} \mathrm{~cm}$, whereas

$$
\begin{aligned}
& r_{\text {initial }}=\left(3 / 8 \pi \rho_{P}\right)^{1 / 2}=5.58 \times 10^{-34} \mathrm{~cm} \\
& \Rightarrow T_{\text {initial }}=2.65 \times 10^{-180} \mathrm{~K}(\mathrm{kelvin}) \\
& r_{D E-\text { formation }}=\breve{a}=10^{-3} \mathrm{~cm} \\
& \Rightarrow T_{D E-\text { formation }}=7.41 \times 10^{31} \mathrm{~K}(\text { kelvin })
\end{aligned}
$$

Is Eq.(36b) going to be feasible as to condtions as to the breakup of black holes, which may produce DE We will be deriving Eq.(36) as a summary of what to expect in this treatment of nonsingular space-time To do so we start off with [2] in pre matter and radiation periods with entropy S, $\rho=\rho(T), P=P(T)$

$$
\begin{aligned}
& d S(V, T)=\frac{1}{T} \cdot[d(\rho V)+P d V] \\
& V=V(\text { volume })=2 \pi^{2} r^{3}
\end{aligned}
$$

And an integratability condition on Eq. (36) leading to

$$
\frac{d P}{d T}=\frac{1}{T} \cdot(\rho+P)
$$

Then the integral of Eq. (37) is given as

$$
S=\frac{V}{T} \cdot(\rho+P)
$$

Also, we look at a given value of pressure as given in [2] for which

$$
P=\frac{\rho}{3} \cdot\left(1-\frac{4 \rho}{\rho_{P}}\right)
$$

Put Eq. (37d) into Eq. (37b) and then one will get after integrating Eq. (37b)

$$
\rho \cdot\left(1-\frac{\rho}{\rho_{P}}\right)^{7}=\sigma T^{4}
$$

Here, [2] treated $\sigma$ as the Stephan-Boltzman constant, and so then if we add in the energy equation

$$
\dot{\rho}+3 \cdot(\dot{r} / r) \cdot(\rho+P)=0
$$

Then we put in Eq. (8) into Eq. Eq. (8b) we obtain

$$
\rho=\breve{a}^{4} \rho_{P} /\left(\breve{a}^{4}+r^{4}\right)
$$

We claim that Eq. (38c) put into Eq. (38a) we wll then obtain Eq. (36)

So what is the problem with the Rosen derivation? And using Temperature? See this one 
$E \approx \frac{d(\operatorname{dim}) k_{B} T}{2}$

Taking this as the defining value at Planck temperature, at say the end of inflation, may lead to a value in some sembalance of $10^{32} \mathrm{Kelvin}$, and from that high temperature it would be hard to ascertain when the separate DE would form. Not impossible, but the procedure outlined as to a breakup of black holes, as done up to a radii of 9 million kilometers at least is not solely dependent upon temperature itself but upon DENSITY. We also assume that at the end of inflation, there is a drop in temperature and an end to acceleration of the speed of the rate of expansion of the universe. of expansion of the universe

But, reference [6] has it that there is a critical density we need to consider, and the author is assuming breakup of black holes, initially, may lead to DE.

Does Rosen with his near Planck Temperature at the end of inflation, as he modeled it have overlap with breaking up of primordial black holes?

It is unclear if this is $t$ he case, or even possible.

6. What Rosen model would give us and not give us as to entropy, the arrow of time and DE

A. We will be able to come up with an initial temperature of $10^{\wedge}-180 \mathrm{Kelvin}$, at a radius of about Planck length, in value. Almost absolute zero

B. The temperature of space-time will be of the order of Planck Temperature after expansion of about $10^{\wedge} 30$ times from the initial nonsingular configuration

C. For making effective use of [2] we will be looking at what is measured after measured after $10^{\wedge}-42$ seconds, which is roughly Planck Time, in this model. I.e.

the convention is that we will be using is that we are starting off, in [2] in what is called the pre-matter radiation transition point, in the history of the universe. We should also keep in mind that $\mathrm{A}$. and $\mathrm{B}$. and $\mathrm{C}$ will allow an arrow of time forming due to the

reasons brought up in [5] whereas we have the following Entropy value of [18][19]

$$
S \sim 3 \cdot\left[1.66 \sqrt{g_{*}}\right]^{2} T^{3}
$$

However, having fidelity with respect to Eq. (18), Eq.(18a), Eq. (19) and Eq. (39) will be difficult.

7. Conclusion, our approach to DE and if it is commensurate with Rosen, and $\mathrm{Ng}^{\prime}$ s infinite quantum statistics?

The Rosen approach, as of section 5, and section 6 satisfies the Arrow of time criteria as given in [5]. We do have though that the way to combine DE with entropy would be to utilize $\mathrm{Ng}$ infinite quantum statistics [20] as far as counting

$$
\mathrm{S} \sim \mathrm{n}
$$

, as $\mathrm{n}$ is a graviton count, which presumably would be increasing with the expansion of the universe. However, this would entail in comparing with and making use of the Hawking statement of the arrow of time as commensurate with the expansion of the universe [21]' and would need that as a Necessary\& sufficient conditon to tie in the Rosen approach with DE. And that may 


\section{be very difficult}

The conclusion the author draws is that any full reconciliation between sort of cosmology presented by Rosen in [2] and the procedure as to how the author is trying to construct DE would be in reconciling the counting procedure of $\mathrm{Ng}$, as used for graviton count with Fay Dowker's causal structure as given in [22]. Once that is done, it may be feasible to state $\mathrm{DE}$ as given by the author, and Rosen's thermal cosmology are commensurate sides to the same animal. In doing so, the author hopes to explore if the Causal structure idea of [21] may be employed to make a linkage between DE, the arrow of time, and Entropy as has been suggested by the author in other publications. In addition, ideas in [23],[24], and [25] should be seriously investigated

Finally and not least the author is well aware of Lagrangian representations of GR, as in [26],[27], and [28]. The author hopes that refinement of the issues given in this text will in the spirit of [29], pp 120-125, allow creating full Lagrangian GR development for a more refined Hamiltonian treatment of GR which moves toward a new synthesis of conserved and non conserved Quantitites which allows for making a linkage between GR and QM from the primordial regime of space - time. Possibly to compliment[30] and later improve upon some of the ideas in Loop quantum gravity [31]. The idea also should be in investigating if this research is commensurate with the ideas of Eternal inflation as given in [32] acknowledgement

This work is supported in part by National Nature Science Foundation of China grant No. 11375279

The author declares that there is no conceivable conflict of interest in this document

\section{References}

1. Andrew Walcott Beckwith, “Using “Enhanced Quantization" to Bound the Cosmological Constant, (for a Bound-on Graviton Mass), by Comparing Two Action Integrals (One Being from General Relativity) at the Start of Inflation", pp 21-36, of "Fundamental Physics and Physics Education Research", editors of Burra G. Sidharth • Jesús Carnicer Murillo • Marisa Michelini • Carmen Perea, Published by Springer Nature Switzerland AG 2021 , in Gewerbestrasse 11, 6330 Cham, Switzerland

2. Rosen N., Israelit M. (1991) A Simple Model of the Universe without Singularities. In: Zichichi A., de Sabbata V., Sánchez N. (eds) Gravitation and Modern Cosmology. Ettore Majorana International Science Series, vol 56. Springer, Boston, MA. https://doi.org/10.1007/978-1-4899-0620-5_14

3. Miao Li (Author), Xiao-Dong Li (Contributor), Shuang Wang (Contributor), Yi Wang (Contributor), “Dark Energy", Peking University Press, World Scientific, Singapore, Republic of Singapore, 2015

4. Novello, M. (2005) The Mass of the Graviton and the Cosmological Constant Puzzle. https://arxiv.org/abs/astro-ph/0504505

5. Gerard 't Hooft', "time, the Arrow of time and Quantum Mechanics", Front. Phys., 29 August 2018 I https://doi.org/10.3389/fphy.2018.00081 
6. Katherine Freeze, Mathew Brown, and William Kinney, "The Phantom Bounce, A new proposal for an oscillating Cosmology", pp 149-156, of "The Arrows of Time, A debate in cosmology", Fundamental theories in physics, Volume 172, with Laura Mercini-Houghton, and Rudy Vaas, editors, Springer Verlag, Heidelberg, Federal Republic of Germany, 2012

7. Calmet, X. Carr, B. Winstanley, E. “Quantum Black holes”, Springer Briefs in Physics, Springer Verlag, New York City, New York, USA, 2014

8. Will, C. M. (1998). "Bounding the mass of the graviton using gravitational-wave observations of inspiralling compact binaries" ,PhysicalReviewD. 57 (4):2061-2068. arXiv:gr-qc/9709011. Bibcode:1998PhRvD..57.2061W. doi:10.1103/ PhysRevD.57.2061. S2CID 41690760.

9. Maggiorie, Michele, " Gravitational waves, Volume 1, theory and Experiment", Oxford University Press, New York City, New York, USA, 2008

10. Paul J. Steinhardt; Neil Turok (2006). "Why the cosmological constant is small and positive". Science. 312 (5777): 1180-1183. arXiv:astro-ph/0605173. Bibcode:2006Sci...312.1180S. doi:10.1126/science.1126231. PMID 16675662 S2CID $141 \overline{78620}$

11. Marcio E. S. Alves, Oswaldo D. Miranda, Jose C. N. de Araujo, "Can Massive Gravity be an alternative to Dark Energy ?", https://arxiv.org/pdf/0907.5190v2.pdf

12. Moniz, Paulo Vargas,"Quantum Cosmology-The Supersymmetric Perspective-Vol.1", Springer Verlag, 2010, Berlin, Federal Republic of Germany

13. A.O.Barvinsky, V.P.Frolov, and A.I.Zelnikov," Wavefunction of a Black Hole and the Dynamical Origin of Entropy", Phys.Rev.D51:1741-1763,1995, https://arxiv.org/abs/gr-qc/9404036

14. Aron C. Wall, "A Survey of Black Hole Thermodynamics", https://arxiv.org/pdf/1804.10610.pdf,

15. Diego Pavon," On the degrees of freedom of a black hole" https://arxiv.org/pdf/2001.05716.pdf

16. E. Kolb, and S. Turner “The Early Universe”, Westview Press, Chicago, USA, 1994

17. Claus Kiefer, David Polarski, Alexei A. Starobinsky, “ENTROPY OF GRAVITONS PRODUCED IN THE EARLY UNIVERSE", Phys.Rev. D62 (2000) 043518, https://arxiv.org/abs/gr-qc/9910065

18. Beckwith, A.W. (2018) Initial Conditions for Defining an Arrow of Time at the Start of Inflation? Journal of High Energy Physics, Gravitation and Cosmology, 4, 787-795. https://doi.org/10.4236/jhepgc.2018.44044

19. E. Kolb, and S. Turner "The Early Universe", Westview Press, Chicago, USA, 1994

20. Ng, Y.J. (2007) Holographic Foam, Dark Energy and Infinite Statistics. Physics Letters B, 657, 10-14. https://doi.org/10.1016/j.physletb.2007.09.052"

21. Hawking, S.W. "Arrow of time in Cosmology", Phys. Rev. D 32, 2489 - Published 15 November 1985

22. Dowker,Fay, "Causal sets and the Deep Structure of space-time", https://arxiv.org/abs/gr-qc/0508109

23. Nathan Rosen," Quantum Mechanics of a Miniuniverse", International Journal of Theoretical Physics, Vol. 32, No. 8, 1993

24. Wang, Qingdi; Zhu, Zhen; Unruh, William G. (2017). "How the huge energy of quantum vacuum gravitates to drive the slow accelerating expansion of the Universe". Physical Review D. 95(10): 103504. arXiv: 1703.00543

25. Ng, Y. Jack, Holographic Foam Cosmology: From the Late to the Early Universe. Symmetry 2021, $13,435$. https://doi.org/10.3390/ sym13030435

26. C. W. Misner, K. S. Thorne, and J. A. Wheeler, Gravitation (Freeman, San Fransisco, 1973).

27. R. M. Wald, General Relativity (University of Chicago, Chicago, 1984).

28. E. Poisson, A Relativist's Toolkit (Cambridge University Press, Cambridge, 2004)

29. Dirac, P.A.M., " Lectures on Quantum Mechanics and Relativistic Field Theory”, Martino Publishing, Mansfield Centre, CT, USA, 2012 
30. http://www.phys.ufl.edu/ det/6607/public html/grNotesVarPrin.pdf

31. Ashtekar, Abhay (2009). "Loop Quantum Cosmology: An Overview". Gen. Rel. Grav. 41 (4): 707-741. arXiv:0812.0177. Bibcode:2009GReGr..41..707A. doi:10.1007/s10714-009-0763-4.

32. Winitzki, Sergi, "Eternal Inflation", World Scientific Publishing Co., Pte. Ltd. Singapore, the Republic of Singapore, 2009 